\title{
Laser-induced isotopic selectivity in the resonance ionization of Os
}

\author{
R.K. Wunderlich ${ }^{1,3}$, I.D. Hutcheon' ${ }^{1}$, G.J. Wasserburg' ${ }^{1}$ and G.A. Blake ${ }^{2}$ \\ Division of Geological \& Planetary Sciences, California Institute of Technology, Pasadena CA 91125 \\ (USA)
}

(Received 7 October 1991)

\begin{abstract}
Isotope selective effects in resonance ionization mass spectrometry (RIMS) pose a potentially serious limitation to the application of this technique to the precise and reproducible measurement of isotope ratios. In order to identify some of the underlying causes of isotope selectivity in RIMS and to establish procedures for minimizing these effects, we investigated laser-induced isotope selectivity in the resonance ionization of Os. A single-color, one-photon resonant ionization scheme was used for several different transitions to produce Os photoions from a thermal atomization source. Variations in Os isotope ratios were studied as a function of laser parameters such as wavelength, bandwidth, power and polarization state. Isotope selectivity is strongly dependent on laser power and wavelength, even when the bandwidth of the laser radiation is much larger than the optical isotope shift. Variations in the ${ }^{190} \mathrm{Os} /{ }^{188} \mathrm{Os}$ ratio of $\approx 20 \%$ for a detuning of $0.8 \mathrm{~cm}^{-1}$ were observed on a transition with a small oscillator strength. Large even-odd isotope selectivity with a $13 \%$ depletion of ${ }^{189} \mathrm{Os}$ was observed on a $\Delta J=+1$ transition at low laser intensity; the odd mass Os isotopes are systematically depleted. For $\Delta J=-1$ and 0 transitions the isotope selectivity was reduced by polarization scrambling and for strongly saturating conditions. A technique employing the wavelength dependence of even-even isotope selectivity as an internal wavelength standard was developed to permit accurate and reproducible wavelength adjustment of the laser radiation. This technique provides control over laser-induced isotope selectivity for single-color ionization and enabled us to obtain reproducible measurements of ${ }^{192} \mathrm{Os} /{ }^{188} \mathrm{Os}$ and ${ }^{189} \mathrm{Os} /{ }^{190} \mathrm{Os}$ ratios in the saturation regime for a $\Delta J=+1$ transition with a precision of better than $0.5 \%$. The application of this wavelengthtuning procedure should significantly improve the quality of RIMS isotope ratio data for many elements.
\end{abstract}

Keywords: osmium; resonance ionization mass spectrometry; isotope selectivity.

Correspondence to: I.D. Hutcheon, Division of Geological and Planetary Sciences 170-25, California Institute of Technology, Pasadena CA 91125, USA.

* Division Contribution \# 5012 (741).

${ }^{1}$ Also at: The Lunatic Asylum of the Charles Arms Laboratory, California Institute of Technology.

${ }^{2}$ Also at: The Beckman Institute, California Institute of Technology.

${ }^{3}$ Present address: Physikalisches Institut, Universität Augsburg, Augsburg, Germany.

0168-1176/92/\$05.00 $\quad$ C 1992 Elsevier Science Publishers B.V. All rights reserved. 


\section{INTRODUCTION}

The application of both thermal ionization mass spectrometry and secondary ion mass spectrometry for the isotopic analysis of important systems in geochemistry, cosmochemistry and materials science is limited by the relatively poor sensitivity of these techniques for elements with high ionization potential, and by isobaric interferences. The development of resonance ionization mass spectrometry (RIMS) has provided a new approach for achieving increased sensitivity and selectivity for a wide range of elements. RIMS has been successfully applied to the analysis of nearly 70 elements. Very high sensitivities in trace elemental analysis have been obtained by combining resonance ionization with ion and laser microprobe techniques [1-3] or with thermal atomization [4-6]. The high efficiency and elemental selectivity [7] make laser resonance ionization a potentially important tool for applications in analytical mass spectrometry.

The determination of isotope ratios by RIMS requires a thorough characterization of laser-induced isotope selective effects. Isotopic selectivity has been reported in RIMS studies of $\mathrm{Mo}, \mathrm{Sn}, \mathrm{Ti}, \mathrm{Os}$ and $\mathrm{Nd}$ using either ion sputtering $[8,9]$ or thermal atomization $[10,11]$ sources. This isotope selectivity, induced by laser excitation and ionization, produces large shifts in measured isotopic ratios and led us to undertake an investigation of the effects of variations in laser parameters such as intensity, bandwidth or polarization state, and in atomic parameters on isotope ratios measured by RIMS. In this study we focus on effects in RIMS measurements of Os isotope ratios because of the importance of the platinum group elements in geochemistry. Isotopic shifts and hyperfine structure in Os are also typical of many elements in this mass range. It was our intention to characterize isotopic effects in Os as a model system, to provide a basis for more general conclusions regarding the use of RIMS in analytical mass spectrometry.

Laser-induced selectivity in RIMS can be divided into three broad categories, summarized below: (i) laser bandwidth and wavelength tuning effects; (ii) selection rule effects; and (iii) dynamic effects.

\section{Bandwidth effects}

In the presence of optical isotope shifts (ISs) and hyperfine structure (HFS), the finite laser bandwidth and accuracy of the laser wavelength setting can produce an incomplete overlap of the laser radiation field with the absorption maxima of different isotopes and their HFS components, frequently giving rise to even-even and even-odd isotope selectivity [12]. Furthermore, since the linewidth of the atomic absorption and the saturation characteristics of individual transitions depend in part on the laser spectral profile, this selectivity will be power dependent. The magnitude of these effects depends in detail 
on the relationship between laser parameters and the IS, HFS and matrix element of a particular transition.

\section{Selection rule and polarization effects}

For a transition between states of angular momentum $J$ and $J^{\prime}$ the fraction of degenerate magnetic sublevels participating in that transition depends, for light of a pure polarization state, on the type of transition, i.e. on whether $\Delta J=-1,0$, or +1 . For $\Delta J=0$ or -1 the fraction of sublevels changes in the presence of nuclear spin owing to the increased ground state degeneracy. This change can produce large variations in the selectivity of the excitationionization process for even vs. odd mass isotopes [13]. In principle, these effects can be calculated and corrected analytically. However, in many experiments the polarization state of the laser is not well characterized and reliable correction factors cannot be obtained. These effects are absent for a $\Delta J=+1$ transition or for unpolarized light.

\section{Dynamic effects}

These effects depend on the detailed time evolution of the interaction between the atomic system and the laser radiation [14-16], including quantum mechanical coherence. Differences in the ionization probabilities for atoms with and without nuclear spin arise from changes in the discrete and continuum matrix elements with the angular momentum coupling scheme, as necessitated by the presence of nuclear spin. A dependence of measured even-odd isotope ratios on laser power, reflecting the competition between ionization rate and HFS coupling time, has been predicted for $\mathrm{Sn}$ under non-saturating conditions [16]. This type of isotope selectivity should disappear under saturating conditions. Recent calculations for the photoionization of $\mathrm{Sn}$ with a $1+1$ ionization scheme using a realistic model for the spatial intensity distribution in the laser beam predict, however, that a finite amount of odd isotope enrichment $(\approx 3 \%)$ should persist in any case owing to the production of photoions in regions where the ionization is not saturated [17]. The magnitude of the enrichment depends on the laser focusing conditions and on the photoionization cross-section of the resonant state.

Precise isotope ratio measurements, such as in the Re-Os system, require the measurement of at least three isotope ratios to correct for mass-dependent isotope fractionation and to determine elemental concentrations by isotope dilution. In addition, the ratios of even to odd mass isotopes, frequently involving a radiogenic isotope, must be measured. The effects described above can significantly influence these ratios and raise the question as to the nature of the constraints on laser parameters such as intensity, bandwidth, polarization 


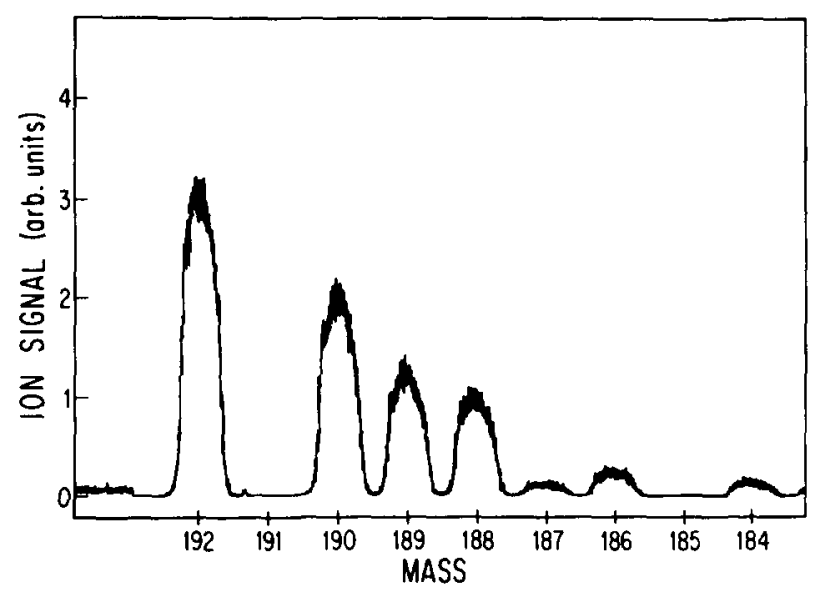

Fig. 2. RIMS mass spectrum of Os with laser (Dye I) tuned to $\Delta J=+1$ transition. Peaks at masses 186 and 184 are due to $W$ (see discussion in the text).

operated like a boxcar integrator. The analog system could be operated at average signal levels as low as 100 ions $\mathrm{s}^{-1}$. However, it was normally used only when the average number of ions generated per laser pulse exceeded 50 at repetition rates of $40-50 \mathrm{~Hz}$.

For the measurement of lower ion currents the multiplier was operated in the pulse counting mode at $3 \mathrm{kV}$ with a gain of $\approx 4 \times 10^{6}$ using a $100 \mathrm{MHz}$ counting system. A critical performance parameter of the counting system is its dynamic range, i.e. the interval over which the number of ions generated per laser pulse can be counted without requiring significant correction for dead time losses in the detector. The dynamic range is determined by the arrivial time distribution of individual ions at the detector and by the bandwidth of the counting system, which is $80 \mathrm{MHz}$ including the multiplier response function. If, for example, the ions generated by a single laser pulse all arrive within a period $f_{c}^{-1}$, where $f c$ is the bandwidth of the counting system, only one ion will be counted per laser pulse. Poisson statistics then imply more than a $30 \%$ dead time correction for an average count rate of 0.5 ions per laser pulse. Other non-linearities affecting the dynamic range of the counting system are a reduction in the multiplier gain owing to space charge effects and current limitations to the dynodes.

Ion arrival time distributions were examined with an oscilloscope. The ion packet generated by a single $15 \mathrm{~ns}$ laser pulse arrives at the detector $100 \mu \mathrm{s}$ after the photodiode trigger. The ion packet is spread out over a time interval of $100 \mu \mathrm{s}$ with the bulk of the ions evenly distributed over the first $50 \mu \mathrm{s}$. This large spread in ion arrival times is due to the finite extent of the ionization volume both parallel and perpendicular to the laser beam and to the low ion 
extraction voltage used in the quadrupole instrument. The arrival time spread of the ions generated by a single laser pulse allows counting of up to 40 individual ions per laser pulse without significant dead time correction. The low ion extraction energy and the finite spatial extent of the ionization volume produce a large increase in the dynamic range of the counting system compared with systems in which only one ion per laser pulse can be counted, and which typically operate at only $\approx 0.3$ ions per laser pulse. The dynamic range of the present pulse counting system is sufficient to allow continuous overlap with the sensitivity range of the analog system. The delay of $100 \mu \mathrm{s}$ between the generation of ions and the arrival of photoions at the detector permitted discrimination between spurious noise counts from electronic transients generated by firing the laser and actual ion events. This type of noise was absent in the analog system. A $13 \mu$ s wide pulse, synchronous with the laser pulse, was used as a veto at the discriminator for noise suppression. Synchronization of the counting time interval with the laser pulse rate is the same as described for the analog system. A timing diagram for the counting system is shown in Fig. 3.

Saturation effects in the counting system were evaluated by examining the dependence of the ${ }^{192} \mathrm{Os} /{ }^{187} \mathrm{Os}$ isotope ratio (nominal value $\approx 25$ ) on the total Os ion signal and by comparing the functional dependence of the ${ }^{192} \mathrm{Os}$ signal on filament temperature measured with both the analog and pulse counting systems at the same filament temperature. No saturation effects were observed for signal levels below 50 ions per laser pulse. Typically, the counting system was operated at a maximum signal level of 40 ions per laser pulse. At a laser repetition rate of $40 \mathrm{~Hz}$ and a signal level of 20 ions per laser pulse and after a careful determination of the multiplier gain with Re thermal ions, we obtained very good agreement between the average number of ions per laser pulse measured with the counting system and the average ion current measured with the analog system. Most experimental results presented here were obtained with the counting system.

Optical spectra were measured with the quadrupole tuned to a specific mass or mass range (integral mode) by recording the variation in ion current as a function of laser tuning. These spectra were used to examine molecular contributions to the ion signal in a specific mass range. The optical spectra also provide a qualitative check on the presence of isotope shifts through a comparison of optical line shapes for a single mass with those obtained for all masses greater than a given mass. In these experiments the multiplier was operated in the analog mode and the ion signal was digitized with a voltageto-frequency converter contained in the Finnigan electronics package. A typical optical ionization spectrum of Os near $39400 \mathrm{~cm}^{-1}$ is shown in Fig. 4 .

The laser system consists of a Lambda Physik LPX $110 \mathrm{iC} \mathrm{XeCl} \mathrm{excimer}$ laser with a maximum repetition rate of $200 \mathrm{~Hz}$ which pumps two Lambda 
Timing Sequence
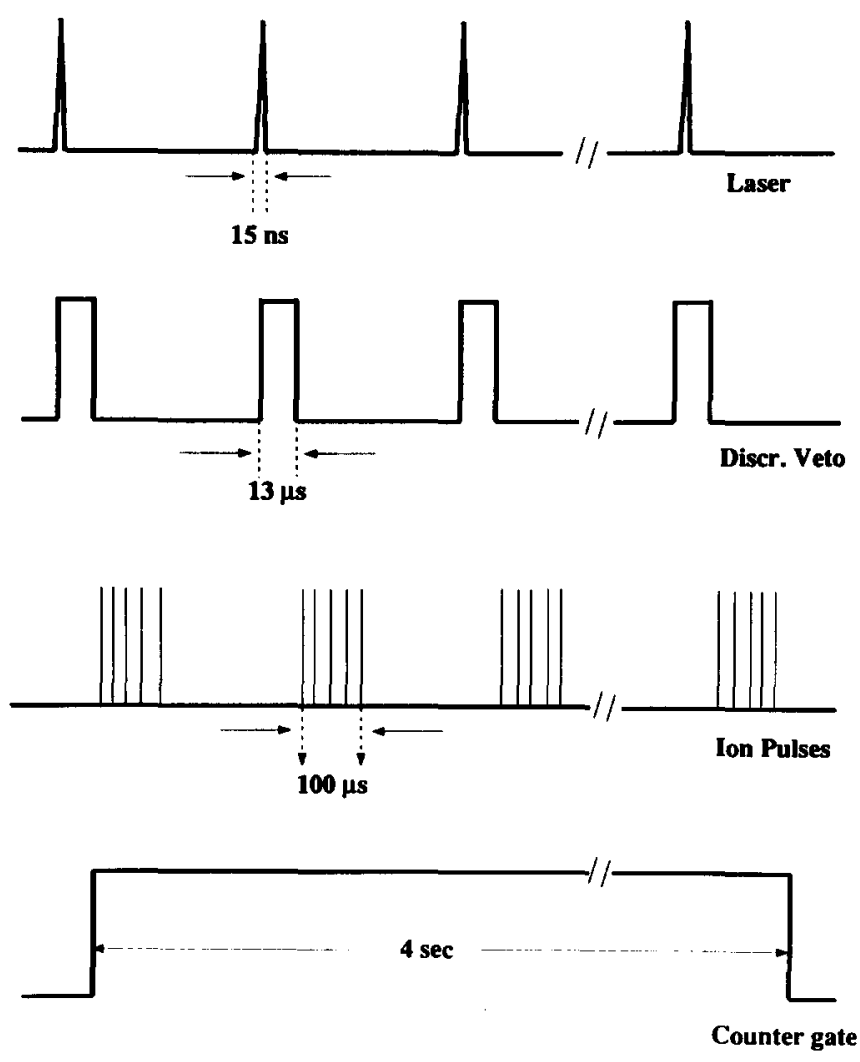

Fig. 3. Timing diagram for the counting system. The counter gate signal is obtained from a precision pulse generator triggered externally by the laser pulse.

Physik FL3002E tunable dye lasers. Tunable radiation in the UV spectral region was generated by frequency doubling the visible dye laser output in KDP and BBO crystals. During the experiments the laser intensity was measured simultaneously with the ion signal using an integrating photodiode and averaged with a DVM over the same integration or counting interval used for the ion current measurement. The reproducibility and pulse-to-pulse stability of the laser wavelength were measured with a pulsed wavemeter to be better than 5 parts per million ( $\mathrm{ppm})$, a value smaller than the typical laser bandwidth $\left(\Delta \omega_{\mathrm{L}}\right)$ of $10 \mathrm{ppm}\left(0.2 \mathrm{~cm}^{-1}\right)$ at $500 \mathrm{~nm}$. For some experiments the fundamental laser bandwidth was increased to $0.37 \mathrm{~cm}^{-1}$ by working in a lower grating order and by slightly misaligning the dye laser oscillator. Alternatively, the lasers could be operated with an intracavity etalon to give a fundamental bandwidth of $0.04 \mathrm{~cm}^{-1}$. No mode structure was observed 


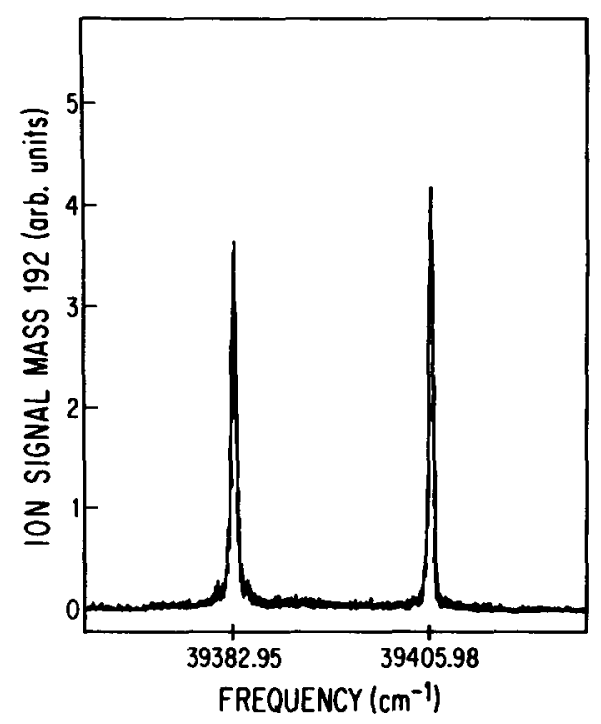

Fig. 4. Optical ionization spectrum of ${ }^{192} \mathrm{Os} ; J=3$ and 5 resonance states.

when the laser was operated in the broad bandwidth mode. During the isotope ratio measurements the laser bandwidth and frequency stability were monitored with an etalon of finesse 18 and a free spectral range of $1.71 \mathrm{~cm}^{-1}$, allowing us to detect changes of the laser wavelength greater than $0.5 \Delta \omega_{\mathrm{L}}$. The wavelength change per step of the grating drive is $0.02 \mathrm{~cm}^{-1}$, compared with a bandwidth of $\Delta \omega_{\mathrm{L}}=0.2 \mathrm{~cm}^{-1}$. We note that even though the reproducibility of the laser wavelength setting was better than $5 \mathrm{ppm}$, larger wavelength shifts were not precluded on a day-to-day basis. Nevertheless, we could measure the dependence of isotope ratios on the fine tuning of the laser wavelength, where "fine tuning" refers to wavelength changes smaller than the laser bandwidth. In the presence of ISs and HFS this is a scale where isotope ratios can be expected to vary significantly with the wavelength of the laser radiation.

\section{Data acquisition and reduction}

Isotope ratios were measured in the peak jumping mode of the THQ. The ion signal at each mass of interest was measured 4-8 times after each mass step with integration or counting times between 1 and $10 \mathrm{~s}$ per measurement, depending on the photoion signal. This mode of data acquisition proved superior to the use of a single, long integration time at each mass because it enabled us to monitor short-period, laser-induced fluctuations in the ion signal. The optimum ratio between integration time and number of measurements of the ion signal at each mass was determined empirically and depended on the overall stability of the system. The laser system was operated at 
$40-50 \mathrm{~Hz}$, since at higher repetition rates laser beam instabilities degraded the measurement precision. Pointing instabilities of the laser beam result from refractive index changes in the dye flow cells owing to heating by the pump laser. This effect was most pronounced for older laser dyes.

In RIMS experiments background signals arising both from non-resonant ionization of molecular and atomic species emitted from the filament and from thermal ions can be expected. In most RIMS experiments the background is evaluated at the mass of interest by detuning the laser off the resonance condition. The absence of any molecular ions in the mass range of $\mathrm{Re}, \mathrm{Os}$ and $\mathrm{Pt}$ in the present RIMS experiments suggests that the background in these experiments was dominated by thermal ions. This was confirmed by the absence of any molecular features in the optical ionization line shape for masses greater than $187 \mathrm{u}$, as shown for Os in Fig. 4. Thus, in the present experiment the thermal ion background was measured at the appropriate non-integer masses with the laser tuned to resonance. In several experiments the background was also measured at the mass of interest with the laser detuned from resonance to verify that the background correction did not introduce systematic error into the isotope ratios. The magnitude of the thermal ion background, expressed as the fractional intensity of the RIMS $\mathrm{Os}^{+}$ion signal, depended on the filament temperature, laser intensity and wavelength. Typically, with a filament temperature of $\approx 1800^{\circ} \mathrm{C}$ and the laser tuned to resonance for Os, the background correction was $1 \%$ at ${ }^{192} \mathrm{Os}, 0.75 \%$ at ${ }^{190} \mathrm{Os}$ and $1.2 \%$ at ${ }^{189} \mathrm{Os}$ and ${ }^{188} \mathrm{Os}$. At the high filament temperatures used in the Os experiments, it is also possible that some of the observed background arose from electron impact ionization, with electrons originating from the acceleration cone of the quadrupole ("Acc" in the inset to Fig. 1).

In the Os measurements a spectrally broad $(\approx 1 \mathrm{~nm})$ background in the region of the $\mathrm{W}$ masses was observed. This background interferes with the measurement of ${ }^{184} \mathrm{Os}$ and ${ }^{186} \mathrm{Os}$. The spectral width of the signal suggests that it did not originate from near-resonant ionization of $\mathrm{W}$ atoms emitted from the filament but rather from photodissociation of W compounds, followed by ionization of excited state $\mathrm{W}$ atoms, similar to the process described below for Pt.

The optical ionization spectrum of Os is markedly different to the ionization line shape obtained for Pt on an Re filament (see Fig. 5) which exhibits molecular features. However, a mass scan over the 184-198 u region, shown in Fig. 6, shows no evidence of peaks other than those due to $\mathrm{Pt}^{+}$. In particular, no signal above noise was measured at the $\operatorname{Re}$ masses $185 \mathrm{u}$ and $187 \mathrm{u}$. The peak in the optical spectra at $40146.5 \mathrm{~cm}^{-1}$ (Fig. 5) is associated with a transition from the thermally populated $J=4\left(E_{J}=823.7 \mathrm{~cm}^{-1}\right)$ level of the ground state electronic configuration to a $J=3$ state lying at $40970.1 \mathrm{~cm}^{-1}$. The absence of any molecular ion peaks in the mass spectrum 


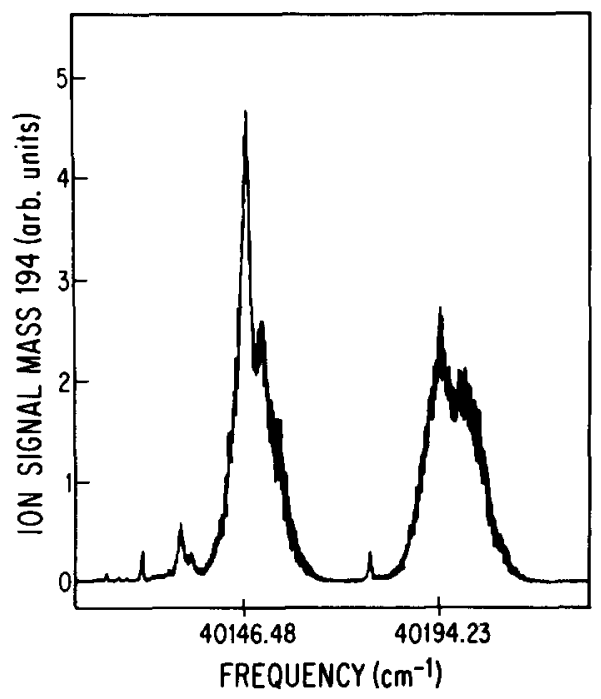

Fig. 5. Optical ionization spectrum of ${ }^{194} \mathrm{Pt}$. The peak at $40146.5 \mathrm{~cm}^{-1}$ arises from the $823.7 \mathrm{~cm}^{-1} J=4$ ground state connected to the $40970.1 \mathrm{~cm}^{-1} J=3$ excited state.

suggests that the molecular features observed in the optical ionization spectrum are due to the photodissociation of $\mathrm{Pt}$ dimers or other $\mathrm{Pt}$ molecules, including clusters, followed by ionization of excited and ground state $\mathrm{Pt}$ atoms. No Pt oxide ions were observed. Similar observations have been reported in RIMS experiments with $\mathrm{Eu}$ and Tc $[18,19]$. No evidence of

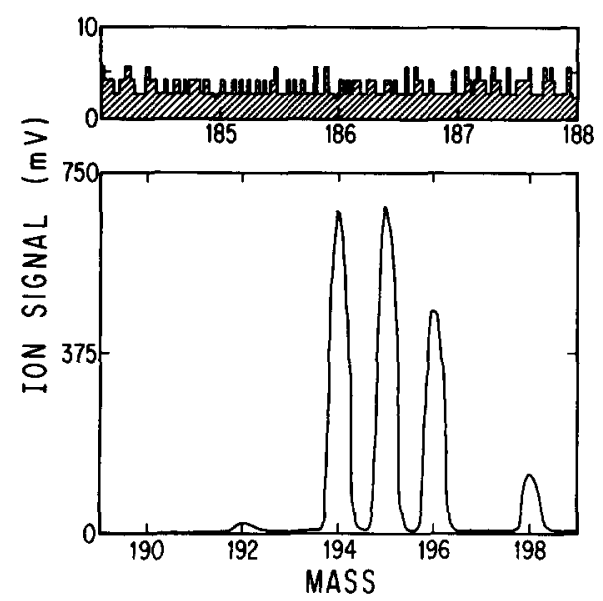

Fig. 6. RIMS mass spectrum of Pt emitted from an Re filament with $\Delta J=+1$ (lower panel). The elemental selectivity of RIMS is demonstrated by the absence of any signal above background in the $\mathrm{Re}$ mass region (upper panel, expanded vertical scale). Laser tuned to $40194.2 \mathrm{~cm}^{-1}$. 
non-resonant ionization of molecular species other than $\mathrm{Pt}_{n}$, or of atoms from the filament material was observed. These observations demonstrate that the elemental selectivity of RIMS with thermal atom sources may depend on the specific combination of sample and filament material and on the chemical reaction dynamics in the evaporation process.

The UV energy per laser pulse, averaged over the counting time at each mass, was used to correct the measured ion signal for laser powerdependent changes in the ion yield. The correction factor was obtained from the ion yield measured as a function of laser intensity. This procedure corrected for drift in the laser intensity during an analysis, while higher-frequency, pulse-to-pulse fluctuations in the laser output averaged to zero over each integration period. Under non-saturating conditions, the application of this correction procedure reduced the error (standard deviation) of blockaveraged isotope ratios by up to $40 \%$ ( $12 \%$ reduced to $8 \%$ ) for some analyses. In no case did this correction increase the standard deviation. A single block consisted of 6-8 individual isotope ratio measurements.

The stability of the RIMS ion signal depended on the degree of saturation of the ionization step, laser beam alignment in the ion source, signal level and dye laser performance. Before each isotope ratio measurement the stability of the ion signal was determined by measuring the $\mathrm{Os}^{+}$intensity for a major isotope $\approx 50$ times with a counting time of $4 \mathrm{~s}$ and a laser repetition rate of $20 \mathrm{~Hz}$. At a signal level of 20 ions per laser pulse and with undersaturating conditions the ratio of the observed fluctuation in the ion current to that expected on the basis of counting statistics was typically 1.2 but increased to 1.4 when the laser power was reduced to half the saturation value. These values agree very well with results obtained with Re thermal ions. An increase in the magnitude of signal fluctuations, observed at an average signal level of 50 ions per laser pulse, indicated the onset of saturation effects in the multiplier. Laser beam alignment can profoundly influence the RIMS signal stability, particularly if the laser beam touches the filament. This is also accompanied by a change in the width of the optical ionization spectrum, providing a means to test for contact of the laser beam with the filament.

The useful yield of the RIMS system was evaluated in an Re RIMS experiment with a standard Re filament as an atom source. The useful yield is defined as the ratio of the number of laser-generated ions per unit time, $N_{\mathrm{L}}^{+}$, to the number of neutral atoms, $N_{0}$, liberated from the filament per unit time. The ion current at the detector is given by $N^{+}=f N_{\mathrm{L}}^{+}$, where $f$ is the instrument transmission, independently measured at $15 \% . N_{0}$ can be calculated from the Clapeyron equation with constants given in ref. 20 and the temperature distribution on the filament, measured with a calibrated optical pyrometer. In this experiment a $100 \mathrm{~cm}$ focal length lens was used for beam focusing, so that the confocal parameter was larger than the longitudinal 
extent of the filament. At a laser repetition rate of $200 \mathrm{~Hz}$ and an intensity of $7 \mathrm{MW} \mathrm{cm}^{-2}$, we obtained a useful yield, $N / N_{0}$ of about $0.1 \%$, and a total efficiency of $10^{-4}$. We estimate that this procedure is accurate to within $\pm 40 \%$. The useful yield can be increased by using a pulsed, rather than a continuously heated filament [21]. For the Os resonance transition $\Delta J=+1$ the efficiency was somewhat lower owing to the smaller photoionization cross-section of the excited state. A precise determination of the useful yield for Os was beyond the scope of these experiments.

\section{RESULTS AND DISCUSSION}

Three different types of experiments were performed, corresponding to common situations in RIMS experiments in which isotope ratios are measured in the presence of both ISs and HFS. First, isotope ratios were measured as a function of laser detuning from resonance to study the sensitivity of RIMS measurements to the exact laser wavelength for a particular transition. These measurements were motivated by the poor reproducibility of isotope ratios in our initial experiments. Knowledge of the wavelength dependence of measured isotope ratios is necessary to distinguish between the different sources of isotope selectivity for a particular transition. Second, isotope ratios were measured as a function of laser intensity to evaluate the power dependence of even-odd isotope selectivity for a $\Delta J=+1$ transition and to study the effects of power broadening on even-even isotope selectivity. In a third set of experiments even-odd selectivity was measured as a function of laser polarization and focusing conditions for transitions where large effects can be expected, i.e. $\Delta J=0$ or -1 . This study was intended to explore the extent to which laser-induced isotope selectivity depends on the parameters of a particular experiment and to determine whether correction factors, pertaining to all odd mass isotopes or to a set of experimental conditions, could be established which would reduce these isotope effects below the experimental error. This approach, if successful, could considerably increase the number of states in a given spectral region amenable for RIMS analysis without uncontrolled isotope selectivity.

For the isotopic studies of Os, transitions near $250 \mathrm{~nm}$ connecting the $5 \mathrm{~d}^{5} 6 \mathrm{~s}^{2}\left({ }^{5} \mathrm{D}_{4}\right)$ ground state with the $J=3,4$ and 5 levels of the excited state configuration $5 \mathrm{~d}^{5} 6 \mathrm{~s} 6 \mathrm{p}$, which have energies of $39382.95 \mathrm{~cm}^{-1}, 40361.92 \mathrm{~cm}^{-1}$ and $39405.98 \mathrm{~cm}^{-1}$ respectively, were chosen. An ionization spectrum of the $J=3,5$ fine structure doublet is shown in Fig. 4. In this wavelength range all platinum group elements can be resonantly ionized with a simple single-color, one-photon $(1+1)$ scheme. The influence of variations in laser parameters bandwidth, power and detuning from exact resonance-on measured isotope ratios is determined by atomic parameters such as oscillator strength, excited 
state hyperfine coupling constants, photoionization cross-sections and optical ISs. The oscillator strength for the $\Delta J=0$ transition is 0.30 [22]. For the $\Delta J=+1$ transition, the oscillator strength can be estimated from a measurement of the power-broadened width of the ionization line shape, using a laser bandwidth of $0.08 \mathrm{~cm}^{-1}$. We obtained a value of 0.004 , making this particular transition rather weak. From the similarity in the power-broadened widths of the $\Delta J=+1$ and -1 transitions, we conclude that both transitions possess similar oscillator strengths. Photoionization cross-sections can be deduced from the saturation behavior of the ionization yield as a function of the laser intensity [23]. A rate equation model with the photoionization cross-section, $\sigma_{\mathrm{I}}$, as a free parameter was used to fit the measured ion yield as a function of laser intensity. In this way we obtained $\sigma_{\mathrm{I}}=2.1 \times 10^{-18} \mathrm{~cm}^{2}$ for the $J=5$ resonance state and $\sigma_{\mathrm{I}}=3.2 \times 10^{-18} \mathrm{~cm}^{2}$ for the $J=4$ resonance state. ${ }^{189}$ Os has a nuclear spin $I=3 / 2$, while $I=1 / 2$ for ${ }^{187}$ Os. The HFS coupling constants of the excited states are not known. An upper limit on the width of the HFS transition array was obtained from a high-resolution $\left(\Delta \omega_{\mathrm{L}}=0.08 \mathrm{~cm}^{-1}\right.$ compared with a Doppler width of $\left.0.11 \mathrm{~cm}^{-1}\right)$, low-laser intensity scan of the ionization line of ${ }^{189} \mathrm{Os}$. We find FWHM widths of $0.39 \mathrm{~cm}^{-1}$ and $0.22 \mathrm{~cm}^{-1}$ for the $\Delta J=+1$ and -1 transitions in ${ }^{189} \mathrm{Os}$ respectively, compared with a width of $0.12 \mathrm{~cm}^{-1}$ for the even-mass isotopes. The optical isotope shifts, $\Delta T$, for the pair ${ }^{190} \mathrm{Os}-{ }^{192} \mathrm{Os}$ are between $0.05 \mathrm{~cm}^{-1}$ and $0.06 \mathrm{~cm}^{-1}$ for these transitions [24] and cannot be resolved in the optical spectra. However, we can obtain an upper limit for the IS by switching the mass setting between the two fine structure components in an optical scan, similar to that shown in Fig. 4. An approximate linear correlation of the IS with mass difference can be expected in Os $[25,26]$; i.e. the center of the ${ }^{189} \mathrm{Os}$ line is located between the ${ }^{188} \mathrm{Os}$ and ${ }^{190} \mathrm{Os}$ lines.

\section{Wavelength dependence}

The variation of the ${ }^{190} \mathrm{Os} /{ }^{188} \mathrm{Os}$ isotope ratio as a function of laser detuning for the $\Delta J=+1$ transition and with saturating (ionization) laser intensity is shown in Fig. 7. This transition has a rather small oscillator strength. However, other sources of isotopic selectivity for this transition are expected to be absent under saturating conditions. The variations in isotope ratios are expressed using $\delta$ notation, which describes the permil deviation (parts per thousand) of a measured isotope ratio, $R_{\mathrm{M}}$, relative to a standard value $R_{\mathrm{S}}$ : $\delta\left(R_{\mathrm{M}}\right)=\left(R_{\mathrm{M}} / R_{\mathrm{S}}-1\right) \times 10^{3}$

Results are shown for two different laser bandwidths, $\Delta \omega_{\mathrm{L}}=0.40 \mathrm{~cm}^{-1}$ and $0.74 \mathrm{~cm}^{-1}$. The steep slope of the curve for $\Delta \omega_{\mathrm{L}}=0.40 \mathrm{~cm}^{-1}$ is unexpected considering that the laser bandwidth is a factor of eight larger than the isotope 


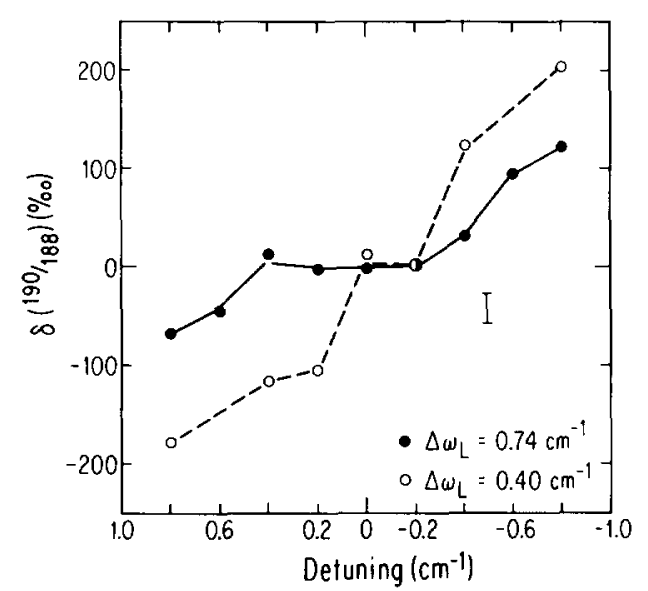

Fig. 7. Variation of the ${ }^{190} \mathrm{Os} /{ }^{188} \mathrm{Os}$ ratio, i.e. even-even isotope selectivity, shown as $\delta^{190} \mathrm{Os}$, as a function of the wavelength of the laser radiation (shown as detuning from resonance) for $\Delta J=+1$ and saturating laser intensity. Open circles represent large laser bandwidth, $\Delta \omega_{\mathrm{L}}=0.74 \mathrm{~cm}^{-1}$, filled circles represent a small bandwidth, $\Delta \omega_{\mathrm{L}}=0.40 \mathrm{~cm}^{-1}$. Bar indicates representative $\pm 1 \sigma$ error.

shift, $\Delta T$, of the pair ${ }^{188}$ Os- ${ }^{190}$ Os [24]. A similar curve was obtained for the variation of the ${ }^{192} \mathrm{Os} /{ }^{190} \mathrm{Os}$ isotope ratio with detuning, showing that $\Delta T$ $(188-190) \approx \Delta T(190-192)$ for this transition. These results demonstrate that unless the laser wavelength is controlled much better than the laser bandwidth, reliable and reproducible isotope ratios cannot be obtained for this particular transition. Similar results were obtained with a larger bandwidth, $\Delta \omega_{\mathrm{L}}=0.74 \mathrm{~cm}^{-1}$; however, the absolute value of the wavelength is somewhat less critical. In particular, the measured ${ }^{190} \mathrm{Os} /{ }^{188} \mathrm{Os}$ ratio is independent of the laser wavelength over an interval of $0.6 \mathrm{~cm}^{-1}$ near resonance. For the $\Delta J=0$ transition at $40361.92 \mathrm{~cm}^{-1}$, which has a similar IS but a larger oscillator strength, the dependence of even-mass isotope selectivity on laser wavelength is much less pronounced. This resonance, however, also exhibits a polarization and power dependence of the even-odd isotope selectivity. The pronounced wavelength dependence found in this experiment raises the question of how one may differentiate between smooth, purely mass-dependent isotope fractionation, which can be corrected analytically by applying a specific fractionation "law" [27,28], and laser wavelengthdependent isotope selectivity for the even-mass isotopes.

The results obtained with the smaller laser bandwidth, $\Delta \omega_{\mathbf{L}}=0.40 \mathrm{~cm}^{-1}$, show that even slight shifts in the laser wavelength, by less than one-half the laser bandwidth, can have a pronounced effect on measured isotope ratios. The magnitude of the variation in $\delta^{190}$ Os poses a potentially serious problem for reproducible RIMS isotope ratio measurements, since changes in the laser 
wavelength by $0.16 \mathrm{~cm}^{-1}$ can hardly be detected in an optogalvanic cell, commonly used for wavelength calibration in RIMS experiments. However, the strong dependence of $\delta^{190}$ Os on wavelength (Fig. 7) suggests that the measurement of even-mass Os isotope ratios can serve as a sensitive internal wavelength standard to adjust the laser wavelength. This technique can be visualized by following the curve in Fig. 7 for $\Delta \omega_{\mathrm{L}}=0.74 \mathrm{~cm}^{-1}$ near line center, where $\Delta\left(\delta^{190} \mathrm{Os}\right) / \Delta \lambda=0$ for several grating steps. The ${ }^{190} \mathrm{Os} /{ }^{188} \mathrm{Os}$ ratio measured in this region is assumed to differ from the absolute ${ }^{190} \mathrm{Os} /{ }^{188} \mathrm{Os}$ ratio in the sample only by mass-dependent fractionation. Once the magnitude of the purely mass-dependent isotope fractionation is established, the laser can be precisely and reproducibly tuned to the proper wavelength after each sample change or laser beam realignment by requiring that the isotopic fractionation remain constant.

The application of this procedure to the measurement of isotope ratios requires that all measured even-mass Os isotope ratios $R_{\mathrm{M}}^{i j}$, are related to the absolute ratios, $R_{\mathrm{S}}^{i j}$, in the sample by a single fractionation law:

$R_{\mathrm{M}}^{i j}(1+\alpha)^{n}=R_{\mathrm{S}}^{i j}$

where $\alpha$ is the fractionation per mass unit and $n=i-j$. Thus, a set of conditions with regard to $\Delta \omega_{\mathrm{L}}$ and laser intensity must be established experimentally for which the value of $\alpha$ is constant for all even-mass isotope pairs, independent of mass, and unaffected by variations in laser detuning and bandwidth. In a RIMS experiment one isotope ratio, for example ${ }^{190} \mathrm{Os} /{ }^{188} \mathrm{Os}$, is used as a pivot element for wavelength tuning until the specified $\alpha$ value has been obtained. All other isotope ratios are then measured without further adjustment of laser parameters.

This technique was evaluated by measuring Os isotope ratios for the $\Delta J=+1$ and 0 transitions with different laser detunings and bandwidths. The results are summarized in Table 1, where we show the values of $(1+\alpha)^{2}$ for different Os isotope ratios. In all experiments the ${ }^{190} \mathrm{Os} /{ }^{188} \mathrm{Os}$ ratio served as an internal standard for wavelength adjustment following each sample change or optical realignment. For zero detuning this procedure produces very good agreement between the $(1+\alpha)^{2}$ values for the different even-mass isotope pairs; the isotope ratio measurements converge to define a mean value, $(1+\alpha)^{2}=1.016 \pm 0.004$. The constancy of the isotopic fractionation for all even-mass isotope pairs indicates control over laser-induced isotopic selectivity substantially exceeding that achieved in previous RIMS studies [8-10]. The agreement between the values of $(1+\alpha)^{2}$ obtained after major realignments of the optical set-up and for several samples indicates that with a suitable choice of experimental parameters, the instrumental massdependent fractionation determined on a standard can be applied to interpret results from samples of unknown isotopic composition. This capability is 


\section{TABLE 1}

Even-mass isotope selectivity in Os for the $\Delta J=0,+1$ transitions as a function of laser detuning and bandwidth. Isotope selectivity is represented by values of $(1+\alpha)^{2}$ (see discussion in the text for a definition of $\alpha$ ). The ${ }^{190} \mathrm{Os} /{ }^{188} \mathrm{O}$ s ratio was used as an internal standard to adjust the laser wavelength in all measurements. For zero detuning, data for all isotope pairs give consistent results, indicating the absence of laser-induced isotope selectivity. For positive detuning the heavier isotopes are depleted. [(1) $\Delta \omega_{\mathrm{L}}=0.40 \mathrm{~cm}^{-1}, \Delta J=+1$; (2) $\Delta \omega_{\mathrm{L}}=$ $0.78 \mathrm{~cm}^{-1}, \Delta J=+1$; (3) $\Delta \omega_{\mathrm{L}}=0.40 \mathrm{~cm}^{-1}, \Delta J=0$ ]

\begin{tabular}{lllll}
\hline Detuning $\left(\mathrm{cm}^{-1}\right)$ & & $(1+\alpha)^{2}$ & \\
\cline { 3 - 5 } & & $190 / 188$ & $192 / 188$ & $192 / 190$ \\
\hline$(1)$ & 0.0 & 1.013 & 1.014 & 1.016 \\
& 0.16 & 1.163 & 1.132 & 1.103 \\
$(2)$ & 0.0 & 1.018 & 1.020 & 1.024 \\
& 0.16 & 1.014 & 1.022 & 1.026 \\
$(3)$ & 0.0 & 1.010 & 1.014 & 1.012 \\
& 0.16 & 1.043 & 1.059 & 1.072 \\
\hline
\end{tabular}

important for the successful application of RIMS to practical isotope ratio measurements. The alternative approach to achieving reproducible isotope ratios, determination of the optical ionization line shape for all isotopes of interest, requires precise measurements over a much larger wavelength interval for each isotope and is impractical for analytical studies.

For the pair ${ }^{192} \mathrm{Os} /{ }^{188} \mathrm{Os}$ we have $\Delta \omega_{\mathrm{L}} / \Delta T(188-192) \approx 4$ for $\Delta \omega_{\mathrm{L}}=$ $0.40 \mathrm{~cm}^{-1}$. For a bandwidth of $0.40 \mathrm{~cm}^{-1}$, the effect of a small laser detuning $\left(<0.16 \mathrm{~cm}^{-1}\right)$ on the even-mass isotope ratios is significant, producing a $10 \%$ depletion of the heavier isotopes for positive detuning (Table 1 (1)). This depletion reflects the increase in the average nuclear charge radius with mass [29], resulting in a shift to lower energies for the heavier Os isotopes. This behavior is reflected in the sign change of $\delta^{190} \mathrm{Os}$ in Fig. 7, where the heavier mass is depleted for positive (energy) detuning. For the $\Delta J=0$ transition, which has a much larger oscillator strength, the effect of laser detuning is less pronounced, although still significant (Table 1 (3)), emphasizing the role of power broadening for the measurement of unbiased even-mass isotope ratios in the presence of ISs. For a larger laser bandwidth, $\Delta \omega_{\mathrm{L}}=0.74 \mathrm{~cm}^{-1}$, the effect of laser detuning cannot be discerned within the experimental precision (Table 1 (2)). The slight change in the value of $(1+\alpha)^{2}$ compared with the value for the $\Delta \omega=0.40 \mathrm{~cm}^{-1}$ experiments is caused by a different laser beam alignment in the ion source. From the ${ }^{190} \mathrm{Os} /{ }^{188} \mathrm{Os}$ ratios measured with $\Delta \omega_{\mathrm{L}}=0.74 \mathrm{~cm}^{-1}$, we obtain a mass fractionation of $\approx 8 \%$ per $u$. All of our experiments show isotopic mass fractionation favoring the lighter isotopes, 


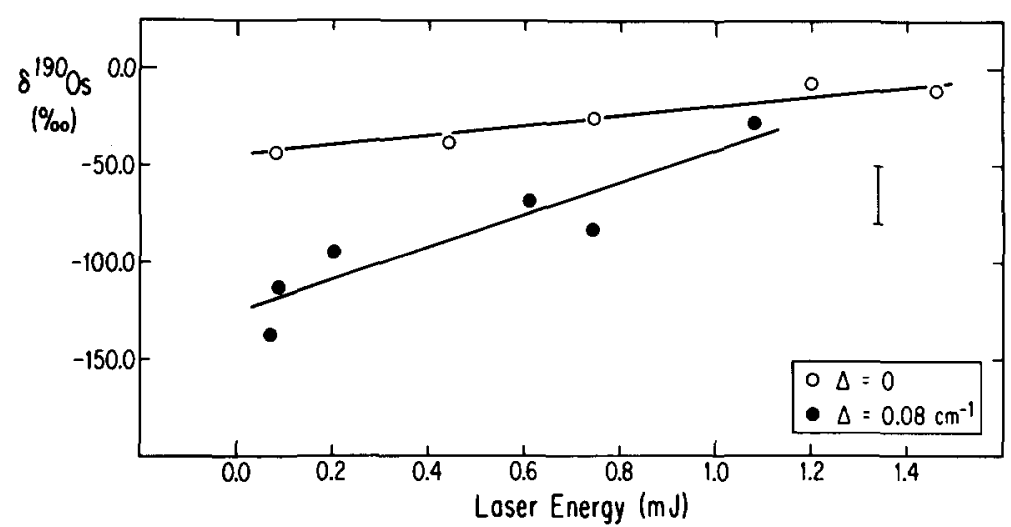

Fig. 8. Variation of the ${ }^{190} \mathrm{Os} /{ }^{188} \mathrm{Os}$ ratio (as $\delta^{190} \mathrm{Os}$ ) as a function of laser energy per pulse; $\Delta J=+1, f=35 \mathrm{~cm}, \Delta \omega_{\mathrm{L}}=0.40 \mathrm{~cm}^{-1}$. Open symbols represent resonant tuning of the laser, filled symbols represent detuning by $+0.08 \mathrm{~cm}^{-1}$.

suggesting that the lower velocities of the heavier isotopes make them more susceptible to fringing fields [30].

The pronounced wavelength dependence of the even-mass isotope selectivity is suggestive of a concomitant power dependence owing to power troadening of spectral lines. Figure 8 shows a plot of the variation in the ${ }^{190} \mathrm{Os} /{ }^{188} \mathrm{Os}$ ratio (shown as $\delta^{190} \mathrm{Os}$ ) as a function of laser power for the $\Delta J=+1$ transition. The upper curve corresponds to zero detuning, $\Delta=0$, the lower curve to positive detuning, $\Delta=+0.08 \mathrm{~cm}^{-1}$. The difference in slope between the two curves again emphasizes the importance of fine tuning of the laser wavelength, especially in the low intensity regime. The depletion of the heavier isotope at low laser power is most likely due to a slight detuning of the laser central wavelength in favor of the lighter isotope. At higher intensities this effect is masked by power broadening, demonstrating the importance of power broadening for the measurement of isotope ratios with moderate bandwidth lasers in the presence of ISs. The dependence of even-mass isotope selectivity on the oscillator strength for finite laser detuning and a given laser bandwidth may be the cause of an apparent discrepancy in Nd isotope ratios measured for different transitions in a RIMS study of $\mathrm{Nd}$ [11], if the laser wavelength was not properly controlled in those experiments.

\section{Dynamic effects}

Dynamic effects in even-odd isotope selectivity were studied by measuring the power dependence of the ${ }^{189} \mathrm{Os} /{ }^{190} \mathrm{Os}$ and ${ }^{189} \mathrm{Os} /{ }^{188} \mathrm{Os}$ ratios for the $\Delta J=+1$ transition, for which selection rule-induced selectivity is absent. This case is similar to the one described by Fairbank et al. [8], who found a $310 \%$ variation in the ${ }^{117} \mathrm{Sn} /{ }^{118} \mathrm{Sn}$ ratio for a $\Delta J=+1$ transition under non- 


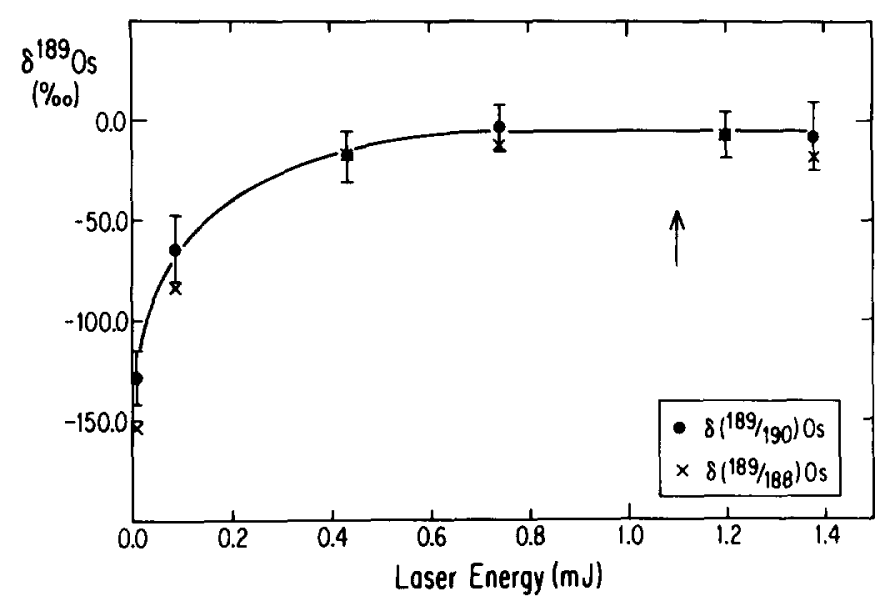

Fig. 9. Variation of the ${ }^{189} \mathrm{Os} /{ }^{190} \mathrm{Os}$ ratio (as $\delta\left({ }^{189} \mathrm{Os} /{ }^{190} \mathrm{Os}\right)$, filled circles) and the ${ }^{189} \mathrm{Os} /{ }^{188} \mathrm{Os}$ ratio (as $\delta\left({ }^{189} \mathrm{Os} /{ }^{188} \mathrm{Os}\right)$, crosses), i.e. even-odd isotope selectivity, as a function of laser energy per pulse; $\Delta J=+1, f=35 \mathrm{~cm}, \Delta \omega_{\mathrm{L}}=0.40 \mathrm{~cm}^{-1}$. Arrow indicates the laser intensity $\left(\approx 1.1 \mathrm{~mJ}\right.$ pulse $\left.{ }^{-1}\right)$ typically used in the RIMS experiments.

saturating conditions. Figure 9 shows the power dependence of the ${ }^{189} \mathrm{Os} /{ }^{188} \mathrm{Os}$ ratio. In Fig. 10 the saturation behavior of the ionization yield for ${ }^{192} \mathrm{Os}$ as a function of laser power, measured under the same experimental conditions, is shown. At low laser intensities ${ }^{189} \mathrm{Os}$ is strongly depleted relative to both ${ }^{188} \mathrm{Os}$ and ${ }^{190}$ Os. The similarity of the depletion in ${ }^{189} \mathrm{Os}$ with regard to both neighboring isotopes suggests that the depletion of ${ }^{189} \mathrm{Os}$ is not caused by laser detuning from resonance. The isotope ratios shown in Fig. 9 were corrected for mass fractionation by the method described above.

These results differ from those obtained for $\mathrm{Sn}$, where an enrichment of the odd mass isotopes under non-saturating conditions was observed [8]. In particular, the value of $\delta^{189} \mathrm{Os}$ is constant over the range of laser intensity from approximately half saturation (ionization) intensity to the maximum intensity used in the experiment. The $\Delta \omega_{\mathrm{L}} / \Delta_{\mathrm{HFS}}$ ratio was very similar in both experiments. However, the more complex HFS and level structure in Os make a direct comparison of the Os and $\mathrm{Sn}$ results difficult. It could be argued that the depletion of ${ }^{189} \mathrm{Os}$ is the result of an incomplete overlap of the laser spectral profile with some components of the HFS transition array, so that these are no longer saturated at the lowest laser power. From a high resolution scan of the ${ }^{189}$ Os line we know that $\Delta \omega_{\mathrm{L}}>\Delta_{\mathrm{HFS}}$. For an HFS component, detuned by $\Delta \omega_{\mathrm{L}}$ from the line center, we find that the discrete transition should be saturated at the lowest intensity used in the experiment for an oscillator strength as low as $\mathbf{0 . 0 0 1}$. Given our estimate of 0.003 for the oscillator 


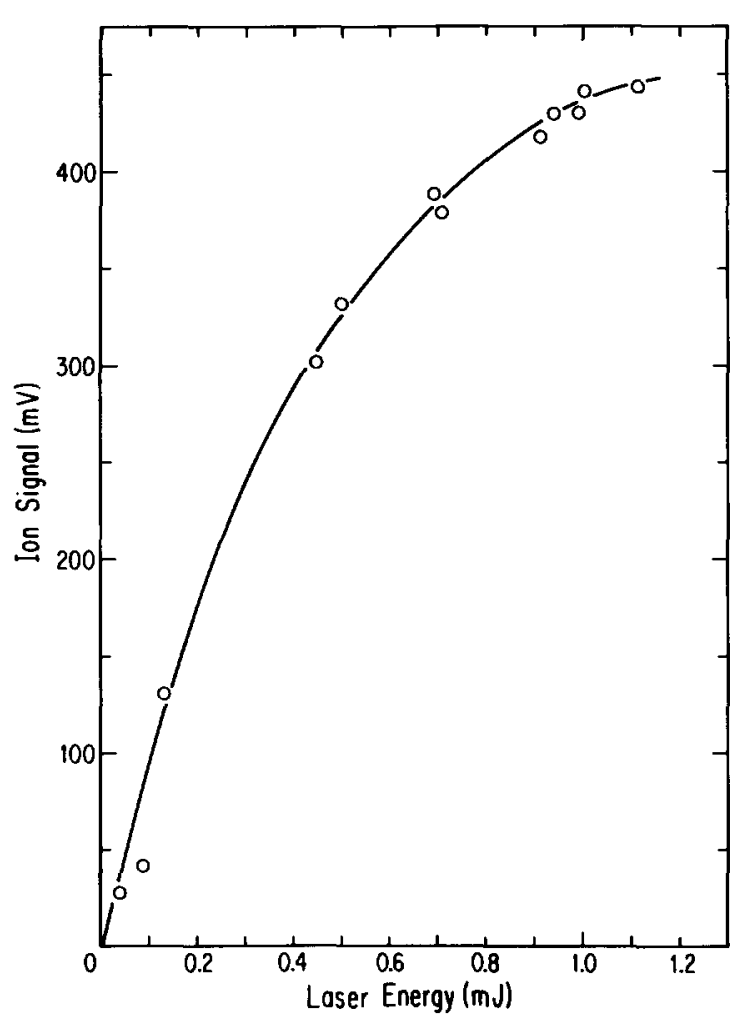

Fig. 10. Ionization yield (number of photoions per laser pulse) for Os as a function of laser energy per pulse. Experimental conditions as in Fig. 8 with $\Delta J=+1$.

strength, we cannot decide if the observed effect is truly "anomalous" [16] or simply related to the finite laser bandwidth.

The results from several different experiments, including major realignments of the lasers and the entire optical set-up, sample changes, and use of different laser bandwidths, all conducted using the ${ }^{190} \mathrm{Os} /{ }^{188} \mathrm{Os}$ ratio for wavelength adjustment, are summarized in Fig. 11. The measurement precision of individual experiments is $0.8-2.0 \%(1 \sigma)$. The results of individual experiments converge to define mean values for the ${ }^{192} \mathrm{Os} /{ }^{188} \mathrm{Os}$ and ${ }^{189} \mathrm{Os} /{ }^{188} \mathrm{Os}$ ratios, ${ }^{190} \mathrm{Os} /{ }^{188} \mathrm{Os}=3.084 \pm 0.011$ and ${ }^{189} \mathrm{Os} /{ }^{188} \mathrm{Os}=0.6098 \pm 0.0030$, that are in excellent agreement with the Os isotope abundances reported by Creaser et al. [31] measured by negative thermal ionization mass spectrometry. Relative to the values in ref. 31 , our data show $\delta^{190} \mathrm{Os}=-0.9 \pm 3.6 \%$ and $\delta^{189} \mathrm{Os}=-3.4 \pm 4.9 \%$. The agreement between our data and the standard ratios indicates that the wavelength tuning technique discussed above can be successfully applied and, at least for the transitions used in this study, permits the determination of both even-even and even-odd Os isotope ratios with a 
$\delta(192 / 188)$

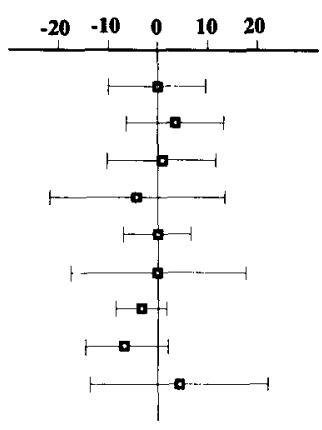

av: $3.0837(0.011)$

$\delta=\mathbf{- 0 . 9 4}$ $\delta(189 / 190)$

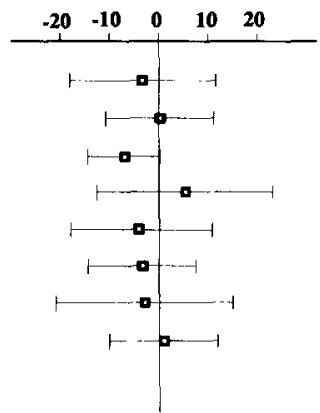

av: $0.6098(0.003)$

$\delta=-\mathbf{3 . 4}$

Fig. 11. Deviations of the ${ }^{192} \mathrm{Os} /{ }^{188} \mathrm{Os}$ and ${ }^{189} \mathrm{Os} /{ }^{190} \mathrm{Os}$ ratios in permil (relative to the values reported by Creaser et al. [31]) measured throughout the course of this study. The data include results from several different experiments, including major realignments of the optical system and the use of several different laser bandwidths. The ${ }^{190} \mathrm{Os} /{ }^{188} \mathrm{Os}$ ratio was used as an internal standard to adjust the laser wavelength for each measurement. Results of the individual measurements converge to define mean values for the ${ }^{192} \mathrm{Os} /{ }^{188} \mathrm{Os}$ and ${ }^{189} \mathrm{Os} /{ }^{190} \mathrm{Os}$ ratios with precisions $(1 \sigma)$ of 3.6 and $4.9 \%$ respectively. The mean isotope ratios are given at the bottom of the figure (av) and the deviations from the values of ref. 31 given in permil. Numbers in parentheses represent $\pm 1 \sigma$ uncertainties.

precision of better than $0.5 \%$. No evidence is found of the $3 \%$ enrichment in ${ }^{189}$ Os reported by Walker and Fassett [10] in a RIMS experiment using a $1+1$ ionization scheme on a different resonance.

\section{Selection rule and polarization effects}

With the assumption that the discrete absorption step is saturated and that the ionization of the excited state is saturated, the isotope selectivity induced by selection rules is easily calculated for light of a pure polarization state. We obtain $\delta^{189} \mathrm{Os}=+215 \%$ and $\delta^{189} \mathrm{Os}=125 \%$ for the $\Delta J=-1$ and $\Delta J=0$ transitions respectively. The enrichment of ${ }^{189} \mathrm{Os}$ (and, more generally, of the odd mass isotopes) is due to the forbidden $M_{J}^{\prime}=0 \rightarrow M_{J}=0$ transition for $\Delta J=0$. However, for the $\Delta J=-1$ transition we observed a much smaller shift, $\delta^{189} \mathrm{Os}=+34 \%$, in our initial experiments. Analysis of the UV light after it had passed through the mass spectrometer ports showed some degree of depolarization, which, when analyzed with a linear polarizer, appeared elliptical with an axis ratio of $P_{\text {ell }}=60$. This depolarization was caused by stress-induced birefringence in the laser ports of the mass spectrometer. Changing the window mounts resulted in an increase of the axis ratio to $\approx 220$. (Stokes parameters were not measured in our experiments.) The notion 
that the light was indeed partially depolarized is inferred from the fact that the measured shift in $\delta^{189} \mathrm{Os}$ is smaller than expected for elliptically polarized light in a $\Delta J=-1$ transition.

The presence of some degree of either elliptical polarization or depolarized light induces additional dynamic effects in the even-odd isotope selectivity, which are absent for light of a pure polarization state. The states of an atom without nuclear spin are represented in the $L S$ coupling scheme by $\left|L S J M_{J}\right\rangle$. In a $\Delta J=-1$ or 0 transition only a fraction, $(2 J-1) /(2 J+1)$ or $2 J /(2 J+1)$ respectively $(\Delta J=0$ applies only for integer $J)$, of the even-mass isotope ground state atoms will participate in the transition driven by linearly polarized light. In the presence of nuclear spin this fraction increases owing to the higher degeneracy, i.e. $(2 J+1)(2 I+1)$ (for broad band excitation HFS states are considered degenerate), of the electronic level, with a resulting enhancement of the odd-mass isotope ion signal. Consider elliptically polarized light, which can be described as a coherent superposition of a major linearly polarized and a minor circularly polarized component. The presence of a $\sigma^{+}$component opens an additional excitation path so that the number of $M_{J}$ states participating in the transition increases. Assume that the ionization step is saturated and non-selective and that no coherence between different $M_{J}$ states is induced. In this case the actual even-odd selectivity in measured isotope ratios depends on the saturation characteristics of the discrete transitions with $\Delta M_{J}=+1$ driven by the $\sigma^{+}$component. Similar arguments apply when the laser beam is described as a superposition of a linearly polarized component with a small degree of depolarized light. In this case, however, the depolarized components can excite both $\Delta M_{J}=+1$ and -1 transitions, compared with light of a coherent elliptical state. This distinction is important for isotopic selectivity in a $\Delta J=-1$ transition, where angular momentum of each handedness must be transferred to the atoms to avoid isotopic selectivity. In contrast, in a $\Delta J=0$ transition with integer $J$, isotopic selectivity can be removed by a coherent superposition of linearly and circularly polarized light. For a focused laser beam with a spatially varying intensity distribution in the ion extraction region, even-odd selectivity will be produced when ions are extracted from regions characterized by a different degree of saturation of the $\Delta M_{J}=+1$ and $\Delta M_{J}=-1$ transitions. The photoionization cross-section determines the amount of ionization contributed from these regions.

In general, even when isotopic selectivity is purely related to selection rules, measured isotope ratios will depend on the polarization characteristics, laser intensity, oscillator strength, focusing conditions, beam divergence, and photoionization cross-section. When the confocal parameter is equal to the spatial extent of the ion extraction region, measured even-odd isotope ratios may depend critically on the laser beam alignment in the ion formation region 
TABLE 2

Even-odd isotope selectivity in Os as a function of laser focusing conditions and the degree of elliptical polarization. Deviations are given in permil relative to ratios reported in ref. 31; numbers in parentheses represent $\pm 1 \sigma$ uncertainties. The ${ }^{190} \mathrm{Os} /{ }^{188} \mathrm{Os}$ ratio was used as an internal standard to adjust the laser wavelength. All of the data for the $\Delta J=-1$ transition were obtained under saturating ionization conditions, while for the $\Delta J=0$ transition the ionization was fully saturated for a focal length $(f)$ of $35 \mathrm{~cm}$ and $\approx 60 \%$ of the saturation value for $f=100 \mathrm{~cm}$. The even-odd isotope selectivity is reduced for less strongly polarized light

\begin{tabular}{|c|c|c|c|c|}
\hline \multirow[t]{2}{*}{$P_{\text {ell }}$} & \multicolumn{2}{|l|}{$f=100 \mathrm{~cm}$} & \multicolumn{2}{|l|}{$f=35 \mathrm{~cm}$} \\
\hline & $\delta(189 / 190)$ & $\delta(189 / 188)$ & $\delta(189 / 190)$ & $\delta(189 / 188)$ \\
\hline \multicolumn{5}{|c|}{$\Delta J=-1$} \\
\hline 220 & $110(19)$ & $115(22)$ & $57(8)$ & $51(12)$ \\
\hline 27 & $74(11)$ & $75(12)$ & $-1.3(13)$ & $5.7(13)$ \\
\hline \multicolumn{5}{|c|}{$\Delta J=0$} \\
\hline 220 & $108(16)$ & $102(14)$ & $89(10)$ & $65(18)$ \\
\hline 27 & $57(16)$ & $41(6)$ & $36(8)$ & $32(8)$ \\
\hline
\end{tabular}

and on the geometry of the ion extraction field [30], particularly if ions are extracted preferentially from regions with lower laser intensity. This condition can produce changes in isotope ratios without inducing significant variations in the total photoion signal. In the case of multi-step ionization schemes and, especially, with non-collinear beam propagation involving $\Delta J=-1$ or 0 transitions [3,9], isotope ratios may also depend critically on the spatial beam overlap of the different lasers, when the ionization laser probes regions with different degrees of saturation of the two components.

In Table 2 we show the variation in ${ }^{189} \mathrm{Os} /{ }^{190} \mathrm{Os}$ ratios (even-odd isotope selectivity) for the $\Delta J=-1$ and 0 transitions, obtained for different degrees of elliptical polarization and focusing conditions; the $\delta^{189}$ Os values are given with regard to both adjacent even-mass isotopes, i.e. $\delta\left({ }^{189} \mathrm{Os} /{ }^{188} \mathrm{Os}\right)$ and $\delta\left({ }^{189} \mathrm{Os} /{ }^{188} \mathrm{Os}\right)$. The experiments were carried out with a laser bandwidth of $\Delta \omega_{\mathrm{L}}=0.66 \mathrm{~cm}^{-1}$, i.e. $\Delta \omega_{\mathrm{L}}>3 \Delta \omega_{\mathrm{HFS}}$. For the $\Delta J=-1$ transition the oddmass isotope enrichment for weak focusing and $P_{\mathrm{ell}}=220$ is approximately half the value expected for light of a pure polarization state. From the symmetry in the depletion of ${ }^{189} \mathrm{Os}$ with regard to ${ }^{188} \mathrm{Os}$ and ${ }^{190} \mathrm{Os}$ and from $\Delta \omega_{\mathrm{L}}>3 \Delta \omega_{\mathrm{HFS}}$, we conclude that laser bandwidth or fine-tuning effects cannot produce the low abundance of ${ }^{189}$ Os compared with the value expected for light of a pure polarization state. Rather, the effects in ${ }^{189}$ Os must be due to the partial saturation of the discrete transitions involving the minor polarization component. The power density in the focal region was $1.5 \times 10^{7} \mathrm{~W} \mathrm{~cm}^{-2}$ and $1.2 \times 10^{8} \mathrm{~W} \mathrm{~cm}^{-2}$ for the $100 \mathrm{~cm}$ and $35 \mathrm{~cm}$ focal length 
lenses respectively. With a higher degree of depolarization and for strong focusing conditions the even-odd selectivity becomes smaller and is reduced below the experimental uncertainty for $P_{\text {ell }}=27$ and a focal length $(f)$ of $35 \mathrm{~cm}$.

The same principal dependence of $\delta^{189}$ Os on polarization and focusing conditions was observed for the $\Delta J=0$ transition. However, owing to the much larger oscillator strength for this transition, it is surprising that for $P_{\text {ell }}=220$ and $f=100 \mathrm{~cm}$ the measured value of $\delta^{189} \mathrm{Os}$ is close to the value expected for light of a pure polarization state. As pointed out above, even a small degree of elliptical polarization should saturate the respective $\Delta M_{J}$ transition and remove the isotopic selectivity. In contrast to the $\Delta J=-1$ transition, for which $\delta^{189} \mathrm{Os} \approx 0$, a finite amount of odd-mass isotope enrichment, $\delta^{189} \mathrm{Os} \approx 34 \%$, remains even under strong focusing conditions.

For the $\Delta J=0$ transition and linearly polarized light, even-odd selectivity arises from the forbidden $M_{J}=0 \rightarrow M_{J}^{\prime}=0$ transition in the even-mass isotopes. This selection rule does not apply for the half-integer total angular momentum states of isotopes with nuclear spin. However, because of the large oscillator strength of this transition a small admixture of circularly polarized light of either handedness (i.e. overall elliptical polarization) should be sufficient to saturate a $\Delta M_{J}=+1$ or -1 transition from the $M_{J}=0$ ground state and thus remove isotopic selectivity on this resonance. Owing to the nature of the frequency doubling process, the UV radiation from the laser is linearly polarized. A small degree of elliptical polarization is induced by the beam steering prisms, if the incoming radiation is not in a pure $p$ or s state with regard to the plane of total reflection. An additional amount of depolarization is induced by passing the laser beam through a quartz window, which is exposed to localized stress.

In order to demonstrate the effect of a small admixture of depolarization to the linearly polarized laser radiation on the even-odd selectivity in a $\Delta J=-1$ or 0 transition, we calculated the spatial distribution of the steady state saturation behavior of two ground state $M$, components (Fig. 12). One component interacts only with the major linearly polarized component, while the other interacts only with the minor component, considered to be an incoherent superposition of left and right circularly polarized light. The saturation of the discrete resonance is expressed as $S(r)=1-n_{0} / n_{\mathrm{tot}}$, where $n_{0}$ is the number density of ground state atoms in the respective $M_{J}$ state and $n_{\text {tot }}$ is the sum of ground and excited state population densities. Saturation of the discrete transition occurs for $S(r)=0.5$. The spatial intensity distribution of the laser beam is represented by a focused gaussian beam with divergence much larger than the diffraction limit. Using a rate equation approximation we also calculated the spatial distribution of the ionization probability, $P_{\mathrm{I}}(r)$, for the two $M_{J}$ ground state components which interact only with the major 

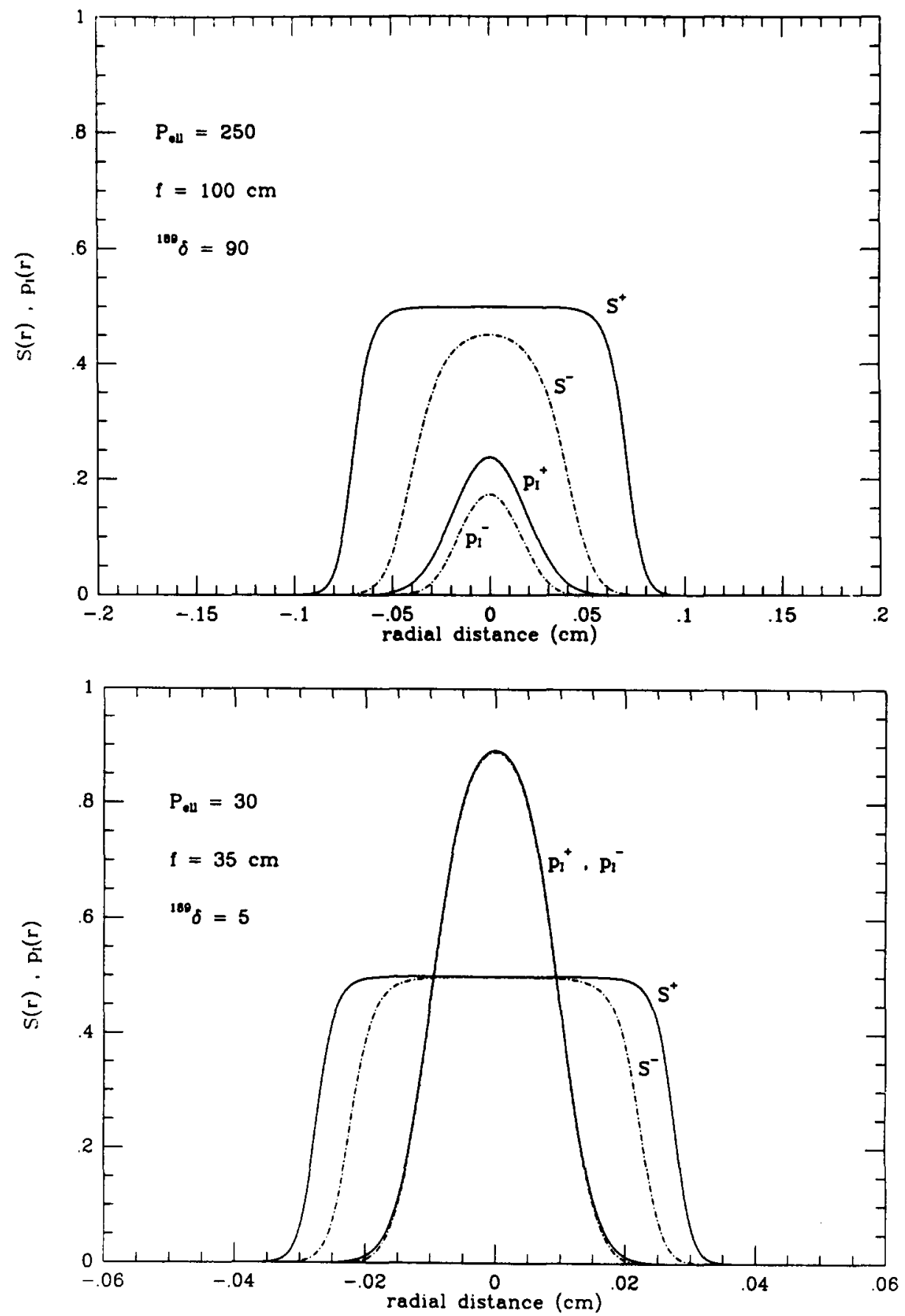

Fig. 12. The radial dependences of the steady state saturation of the discrete transition, $S(r)$, and of the ionization probability, $P_{1}(r)$, for two $M_{J}$ components of the $\Delta J=-1$ transition, calculated for parameters matching experimental conditions. The top panel shows results for weak focusing and strong linear polarization, while the bottom panel shows results for strong focusing and weak polarization. The plus and minus superscripts refer to the major and minor polarization components of the laser radiation respectively. For strong focusing, $S^{+}(r) \approx$ $S^{-}(r) \approx 0.5$ and only a small odd-mass isotope selectivity is predicted, $\delta^{189} \mathrm{Os}=5 \%$. In contrast, for weak focusing $S^{+}(r)>S^{-}(r)$ and the isotope selectivity is much larger, $\delta^{189} \mathrm{Os}=90 \%$. 
and minor polarization components (see Appendix). A similar approach has been used by several authors for a quantitative description of ion yields in resonance ionization, see e.g. ref. 32. The validity of this approach, as compared with an integration of the quantum mechanical equation of motion, has been discussed in several studies [33,34]. It has recently been shown that under the conditions (i) $\Delta \omega_{\mathrm{L}}>\Delta \omega_{\mathrm{HFS}}$ and (ii) $t_{\mathrm{p}}>\Delta \omega_{\mathrm{HFS}}^{-1}$, ion yields calculated in a rate equation analysis agree very well (within $0.2 \%$ ) with results obtained from a numerical integration of the equations of motion [17]. If the discrete transition is strongly power-broadened, as is the case of the experiments considered here, the agreement is less good but discrepancies are less than a few percent. For the same reason, i.e. $\Delta \omega_{\mathrm{L}}>\Delta \omega_{\mathrm{HFS}}$, we do not assume that population trapping caused by ground state hyperfine coherences [35] should influence even-odd isotope ratios. In our model we assume that the interaction of the minor polarization component with states driven by the major component can be neglected and that all excited state sublevels are ionized by the major components with the same efficiency. The total amount of ionization obtained by integrating $P_{\mathrm{I}}(r)$ and summing over all $M_{J}$ ground states is compared with that obtained for an isotope with nuclear spin and similar photoionization cross-section. It should be emphasized that $\delta$ values obtained in this way reflect only the influence of the saturation behavior of the discrete $\Delta M_{J}$ transitions driven by the major and minor polarization components on the isotopic selectivity.

The results of this calculation for the $\Delta J=-1$ transition are shown in Fig. 12 for two cases: (a) weak focusing and a high degree of linear polarization; and (b) strong focusing and a high degree of depolarization. The spatial dependence of the saturation of the discrete transition is shown on the same ordinate as the ionization probability. The pronounced difference in the radial dependence of $S^{+}(r)$ and $S^{-}(r)$ indicates that the isotopic composition of the excited state population varies across the laser beam profile, so that measured isotope ratios will depend critically on laser beam overlap if different lasers are used for excitation and ionization. (The plus and minus signs represent transitions driven by the major and minor polarization components respectively.) In addition, given the finite extent of the neutral atom cloud, even-odd isotope ratios may also depend on the laser beam alignment in the source region. In the strong focusing case and for $P_{\text {ell }}=30, S^{+}(r)=$ $S^{-}(r)=0.5$ over a wide central portion of the laser beam; only a small amount of odd-mass isotope enrichment is produced in the wings of the spatial ionization distribution. The photoionization cross-section determines the amount of ionization contributed from regions where $S^{-}(r)$ is less than $S^{+}(r)$ and, thus, the odd isotope enrichment. For the $\Delta J=-1$ transition the agreement between the $\delta^{189} \mathrm{Os}$ values calculated for $f=35 \mathrm{~cm}, P_{\text {ell }}=250$ and $P_{\text {ell }}=30$, i.e. $\delta^{189} \mathrm{Os}=90 \%$ and $\delta^{189} \mathrm{Os}=5 \%$ respectively, and the values 


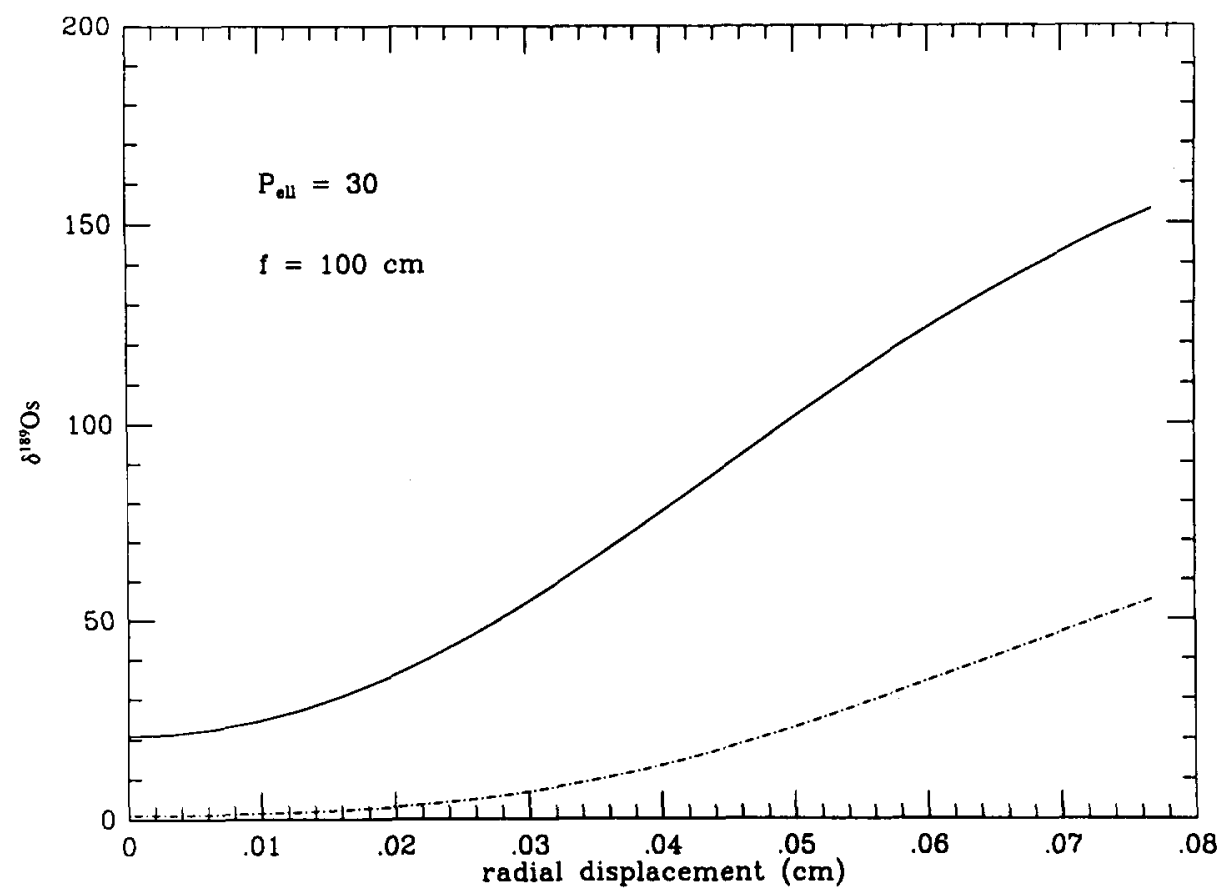

Fig. 13. The variation of $\delta^{189} \mathrm{Os}$ as a function of the displacement of the position of the ionization laser beam, calculated for weak focusing and little polarization for the $\Delta J=-1$ (solid line) and $\Delta J=0$ (broken line) transitions. Significant shifts in $\delta^{189}$ Os occur for displacements of only $0.2 \mathrm{~mm}$ for the $\Delta J=-1$ transition. Central intensity of laser radiation: $2.5 \times 10^{7} \mathrm{~W} \mathrm{~cm}^{-2}$.

actually measured under similar conditions, i.e. $\delta^{189} \mathrm{Os}=110 \%$ and $\delta^{189} \mathrm{Os}=5 \%$, is rather good, and we conclude that the model calculations reflect the experimentally observed trend. However, for the $\Delta J=0$ transition we were unable to reproduce the observed isotopic selectivity for an oscillator strength of 0.3 , even when other input parameters such as $P_{\text {ell }}, \sigma_{1}$ and laser power were varied over a large range. For parameters relevant to our experiments, these calculations predict that the odd-mass isotope enrichment should be less than $1 \%$ for strong focusing and $P_{\text {ell }}=30$. In contrast, the experimentally measured value is ${ }^{189} \mathrm{Os}=34 \%$. The $\Delta J=0$ transition thus exhibits an odd-mass isotope enrichment which cannot be explained within the framework of our rate equation model, assuming equal photoionization cross-sections for even and odd mass isotopes.

We have also used this model to evaluate the dependence of even-odd isotope selectivity on laser beam overlap for experiments in which different lasers are used for the discrete excitation and ionization steps. Results are shown in Fig. 13, as $\delta^{189} \mathrm{Os}$, for the $\Delta J=-1$ and 0 transitions, weak focusing 
and $P_{\text {ell }}=30$. The results emphasize the importance of laser beam alignment for the measurement of reproducible isotope ratios in multi-step ionization schemes involving a $\Delta J=-1$ or 0 transition. Under conditions of strong focusing and a sufficient degree of depolarization, i.e. $I_{\text {Pol }} / I_{\text {Unpol }}<20$, these results suggest that isotopic selectivity can be reduced below the $1 \%$ level, even for transitions with a small oscillator strength. If this projection can be confirmed experimentally, the number of suitable transitions in a given wavelength region for multi-element isotopic analysis using RIMS will be substantially increased.

\section{SUMMARY}

Using RIMS, the wavelength, power and polarization dependence of laserinduced isotopic selectivity has been studied for three transitions in Os. The even-even isotopic selectivity is strongly dependent on laser wavelength and intensity, even when the laser bandwidth is a factor of eight larger than the optical isotope shifts on a transition with a small, i.e. $<0.01$, oscillator strength. These effects can be overcome by fine tuning the laser wavelength to produce a given even-even isotope ratio, which is related to the true ratio by massdependent fractionation. For transitions with a larger oscillator strength wavelength fine tuning is not as critical. However, in practical applications the choice of the transition is often dictated by the possible presence of other sources of laser-induced selectivity and large oscillator strength transitions may not be accessible. Even-even and even-odd isotope selectivities increase for decreasing laser power; for the latter, a depletion of the odd-mass isotope is consistently observed. These isotope shifts affect the reliability and reproducibility of isotope ratios measured at low laser intensities and emphasize the importance of power broadening for the measurement of isotope ratios by RIMS in the presence of optical isotope shifts.

Many RIMS experiments for elements relevant to geochemical or cosmochemical applications employ multi-step ionization schemes because of the higher selectivity and lower laser intensity required to saturate the ionization. Our results, however, show that in the low intensity regime large isotopic effects affecting even-even as well as even-odd isotope ratios can be expected for moderate bandwidth lasers and transitions having a small oscillator strength. For $\Delta J=0$ and -1 transitions the observed even-odd selectivity depends strongly on the polarization characteristics of the laser radiation, focusing and ion extraction conditions, imposing limits on the reproducibility of isotope ratios. Owing to the dynamic nature of these effects it will be very difficult to obtain reliable correction factors for these transitions. However, some of our data suggest that under highly saturating conditions and with 
optimization of the ion extraction optics many isotope shifts can be reduced below the $0.5 \%$ level.

An empirical wavelength tuning procedure, in which the laser wavelength is adjusted to yield a constant ${ }^{190} \mathrm{Os} /{ }^{188} \mathrm{Os}$ ratio, was developed in this study and provides control over laser-induced isotope selectivity superior to that exhibited by previous RIMS experiments. This procedure was successfully applied to determine both even-even and odd-even Os isotope ratios for a $\Delta J=+1$ transition with a reproducibility and accuracy of better than $0.5 \%$. Both the ${ }^{192} \mathrm{Os} /{ }^{188} \mathrm{Os}$ and ${ }^{189} \mathrm{Os} /{ }^{188} \mathrm{Os}$ ratios measured in this study by RIMS are in excellent agreement with published values [31]. While the absolute accuracy of measured isotope ratios depends on the details of the experimental set-up and is sensitive to laser parameters, it appears that the wavelength adjustment technique is generally applicable and should provide a significant improvement in the quality of isotope data acquired by RIMS.

Model calculations, following a rate equation approach, reflect the experimentally observed trends in the laser-induced isotope shifts and enable us to identify sources of isotopic selectivity in RIMS studies with broad band laser systems. The model calculations underscore the complexity of accurate isotope ratio measurements by RIMS and also provide a possible explanation for some of the isotopic effects reported in previous RIMS studies. This area has not been fully appreciated in most RIMS work and it seems worthwhile to extend these studies to more transitions having a wider range of matrix elements to investigate quantitatively the relationship between power broadening and the wavelength dependence of even-even isotope ratios in the presence of a finite optical isotope shift, and to separate more clearly laser power-dependent bandwidth effects from the more fundamental dynamic effects.

\section{ACKNOWLEDGMENTS}

We would like to thank D.A. Papanastassiou for assistance in the initial phase of the experiment and K. Habfast and Finnigan-MAT for aid in obtaining the THQ. Stimulating discussions with M.G. Payne, R.E. Baumgarner and P.G. Green are appreciated. We thank D. Tomlinson and M.E. Johnson for manuscript preparation. This work was supported by DOE grant DE FG03-88ER-13851 and NSF grant EAR-8816936 to G.J. Wasserburg. The laser system used in this project was obtained through support from the Packard and Sloan Foundations and NASA grant NAGW1955 (to G.A.B.).

\section{REFERENCES}

1 J.E. Parks, N.T. Spaar, D.W. Beekman and L.J. Moore, Inst. Phys. Conf. Ser., 94 (1988) 197. 
2 D.L. Pappas, D.M. Hrubowchak, M.H. Ervin and N. Winograd, Science, 243 (1989) 64.

3 J.B. Blum, M.J. Pellin, W.F. Calaway, C.E. Young, D.M. Gruen, I.D. Hutcheon and G.J. Wasserburg, Anal. Chem., 62 (1990) 209.

4 U. Krönert, J. Bown, H.J. Kluge, W. Rusker, K. Wallmeroth, D. Penser and N. Trautmann, Appl. Phys., B38 (1985) 65.

5 G.I. Bekov, V.S. Letokhov and U.N. Radaev, J. Opt. Soc. Am., B2 (1985) 1554.

6 S.V. Andreev, V.I. Mishin and V.S. Letokov, Opt. Commun., 57 (1986) 317.

7 G.S. Hurst, N.G. Payne, M.H. Nayfeh, J.P. Judish and E.B. Wagner, Phys. Rev. Lett., 35 (1975) 82.

8 W.M. Fairbank, Jr., M.T. Spaar, J.E. Parks and J.M.R. Hutchinson, Phys. Rev. A, 40 (1989) 2195.

9 D.R. Spiegel, M.J. Pellin, W.F. Calaway, J.W. Burnett, S.R. Coon, C.E. Young, D.M. Gruen and R.N. Clayton, Proc. Lunar and Planet. Sci. Conf., 22 (1991) 1303.

10 R.J. Walker and J.D. Fassett, Anal. Chem., 58 (1986) 2393.

11 Y.P. Young, R.W. Shaw, D.E. Goeringer and D.H. Smith, Inst. Phys. Conf. Ser., 94 (1988) 367.

12 C.M. Miller, B.L. Fearey, B.A. Palmer and N.S. Nogar, Inst. Phys. Conf. Ser., 94 (1988) 297.

13 C. Hayman, B. Comaskey, M. Johnson and E. Worden, SPIE Proc., 912 (1988) 200.

14 A.T. Georges and P. Lambropoulos, Phys. Rev. A, 18 (1978) 1072.

15 G. Leuchs, S.J. Smith, E. Khawaja and H. Walther, Opt. Commun., 31 (1979) 313.

16 P. Lambropoulos and A. Lyras, Phys. Rev. A, 40 (1989) 2199.

17 M.G. Payne, S.L. Allman and P.E. Parks, Spectrochim. Acta, Part B, 46 (1991) 1439.

18 J.P. Young, D.L. Donohue and D.H. Smith, Int. J. Mass Spectrom. Ion Processes, 56 (1984) 307.

19 S.W. Downey, N.S. Nogar and C.M. Miller, Int. J. Mass Spectrom. Ion Processes, 61 (1984) 337.

20 S.B. Dushman, Scientific Foundations of Vacuum Technique, Wiley, New York, 1962.

21 J.D. Fassett, L.J. Moore, R.W. Shideler and J.C. Travis, Anal. Chem., 56 (1984) 203.

22 U.S. Department of Commerce, Experimental Transition Probabilities for Spectral Lines of 70 Elements, Natl. Bur. Stand. (U.S.), Monogr., 53 (1962).

23 R.K. Wunderlich and T. Kirsten, Chem. Phys. Lett., 106 (1984) 242.

24 P. Aufmuth and E. Wöbker, Z. Phys. A, 321 (1985) 65. A comprehensive listing of IS data can also be found in: Gmelin Handbook of Inorganic Chemistry, System No. 68: Platinum, Springer-Verlag, New York, 1989.

25 A.P. Hines and J.S. Ross, Phys. Rev., 126 (1962) 2105.

26 G. Nöldeke, A. Steudel, K.E. Wallach and H. Walther, Z. Phys., 170 (1962) 22.

27 G.J. Wasserburg, J.B. Jacobsen, D.J. DePaolo, M.T. Culloch and T. Wen, Geochim. Cosmochim. Acta, 45 (1951) 2311.

28 S.A. Hart and A. Zindler, Int. J. Mass Spectrom. Ion Processes, 89 (1989) 282.

29 M.V. Hoehn and E.B. Shera, Phys. Rev. C, 20 (1979) 1934.

30 W.E. Austin, A.E. Holme and J.H. Leck, in P.H. Dawson (Ed.), Quadrupole Mass Spectrometry and its Applications, Elsevier, Amsterdam, 1976.

31 R.A. Creaser, D.A. Papanastassiou and G.J. Wasserburg, Geochim. Cosmochim. Acta, 55 (1991) 597.

32 C.M. Miller and N.S. Nogar, Anal. Chem., 55 (1983) 481.

33 S. Swain, J. Phys. B, 12 (1979) 1231.

34 G.S. Hurst, M.G. Payne, S.D. Kramer and J.P. Young, Res. Mod. Phys., 51 (1979) 767.

35 W.B. Whitten and J.M. Ramsey, Appl. Spectrosc., 44 (1990) 1188. 
APPENDIX: CALCULATION OF ODD-EVEN ISOTOPE SELECTIVITY RELATED TO SELECTION RULES

The number of ions generated for the even- and odd-mass isotopes during a single laser pulse can be expressed as

$N_{\text {even }}^{+}=\frac{n_{\text {even }}^{0}}{2 J+1} \sum_{M_{J}} V_{\mathrm{I}}\left(M_{J}\right)$

and

$N_{\text {odd }}^{+}=\frac{n_{\text {odd }}^{0}}{(2 J+1)(2 I+1)} \sum_{F, M_{F}} V_{\mathrm{I}}\left(M_{F}\right)$

where $n_{\text {even }}^{0}$ and $n_{\text {odd }}^{0}$ are the neutral ground state number densities of the evenand odd-mass isotopes respectively, $J$ is the ground state angular momentum for $I=0$ and $F=J+I . V_{\mathrm{I}}(M)$ is the ionization volume associated with a particular $M_{J}$ or $M_{F}$ component of the ground state electronic configuration and is given in cylindrical geometry by

$V_{\mathrm{I}}\left(M_{J}\right)=\Delta z \int_{0}^{\pi} \int_{0}^{\infty} P_{\mathrm{I}}(r, \phi) \mathrm{d} \phi r \mathrm{~d} r$

where $P_{\mathrm{I}}(r, \phi)$ is the ionization probability of the particular $M_{J}$ component and $\Delta z$ is the longitudinal extent of the region over which ions are collected. We assume that $n^{0}=$ constant for $P_{\mathrm{I}}(r, \phi) \geqslant 0$.

Consider a $1+1$ resonant photoionization scheme and laser radiation consisting of a major, $I^{+}$, and a minor, $I^{-}$, polarization component, e.g. elliptically polarized light for a coherent superposition or some degree of depolarization for an incoherent superposition. We assume $I^{+}>I^{-}$and that the interaction with $I^{-}$can be neglected for $M_{J}, M_{F}$ states which have a non-zero dipole matrix element for transitions driven by the $I^{+}$polarization component. For a $\Delta J=-1$ or 0 transition:

$N_{\text {even }}^{+}=n_{\text {even }}^{0} \frac{1}{2 J+1}\left[\sum_{M_{J}^{+}} V_{1}\left(M_{J}^{+}\right)+\sum_{M_{J}^{-}} V_{\mathrm{I}}\left(M_{J}^{-}\right)\right]$

where $V_{\mathrm{I}}\left(M_{J}^{-}\right)$is the ionization volume of those ground state components interacting only with $I^{-}$. Assume that all excited state components are ionized with the same efficiency by the $I^{+}$component. In this case the difference between $V_{\mathrm{I}}\left(M_{J}^{+}\right)$and $V_{\mathrm{I}}\left(M_{J}^{-}\right)$originates only from the spatial variation in the saturation of the discrete transitions driven by $I^{+}$and $I^{-}$. Consider the ratio

$r=V_{\mathrm{I}}\left(M_{J}^{-}\right) / V_{\mathrm{I}}^{+}\left(M_{J}^{-}\right)$

where $V_{\mathrm{I}}^{+}\left(M_{J}^{-}\right)$is obtained from $V_{\mathrm{I}}\left(M_{J}^{-}\right)$by replacing $I^{+}$by $I^{-}$in the calculation of $P_{\mathrm{I}}(r, \phi)$. Assume that there are $k_{\text {even }}$ and $k_{\text {odd }}$ states which 
interact only with the $I^{-}$component of the laser radiation field, that the individual ionization volumes can be replaced by an average ionization volume, $V_{\text {even }}^{+}$and $V_{\text {odd }}^{+}$, i.e $V_{\mathrm{I}}\left(M_{J}\right)=c\left(M_{J}\right) V_{\text {even }}^{+}$, and that

$\sum_{2 J+1-k} c\left(M_{J}\right) \approx 2 J+1-k$

With these assumptions we obtain:

$N_{\text {even }}^{+}=n_{\text {even }}^{0} \frac{1}{2 J+1}\left[2 J+1-k_{\text {even }}\left(1-r_{\text {even }}\right)\right] V_{\text {even }}^{+}$

and

$N_{\text {odd }}^{+}=n_{\text {odd }}^{0} \frac{1}{(2 J+1)(2 I+1)}\left[(2 J+1)(2 I+1)-k_{\text {odd }}\left(1-r_{\text {odd }}\right)\right] V_{\text {odd }}^{+}$

where similar arguments have been made for the $F_{1} M_{F}$ states.

Define the odd-even $\delta$ value as

$\delta^{\text {odd }}=(R-1) \times 10^{3}$

with

$R=\frac{N_{\text {odd }}^{+}}{N_{\text {even }}^{+}} \cdot \frac{n_{\text {even }}^{0}}{n_{\text {odd }}^{0}}$

or

$R=\frac{\left[(2 J+1)(2 I+1)-k_{\text {odd }}\left(1-r_{\text {odd }}\right)\right] V_{\text {odd }}^{+}}{(2 I+1)\left[2 J+1-k_{\text {even }}\left(1-r_{\text {even }}\right)\right] V_{\text {even }}^{+}}$

The numbers $k_{\text {odd }}$ and $k_{\text {even }}$ are easily obtained from selection rules. For linearly polarized light in a $\Delta J=-1$ transition we obtain $k_{\text {even }}=k_{\text {odd }}=2$ and for $\Delta J=0$ and integer $J$, we obtain $k_{\text {even }}=1$ and $k_{\text {odd }}=0$. The $r$ values are calculated from a rate equation approximation. In a $1+1$ ionization scheme the ionization probability $P_{\mathrm{I}}(r, \phi)$ is given by

$P_{\mathrm{I}}(r, \phi)=R_{\mathrm{I}} \int_{0}^{t_{P}} P_{\mathrm{I}}(t) \mathrm{d} t$

where $P_{\mathrm{I}}(t)$ is the probability for an atom to be in the excited state and is given by

$P_{\mathrm{I}}(t)=\frac{R_{01}}{A} \cdot \exp \left(\frac{1}{2} B t\right) \cdot \sinh \left(\frac{1}{2} A t\right)$

where

$A=\left[\left(2 R_{01}+R_{\mathrm{I}}+A_{10}\right)^{2}-4 R_{01} R_{\mathrm{I}}\right]^{1 / 2}$ 
and

$B=2 R_{01}+R_{\mathrm{I}}+A_{10}$

$R_{01}$ is the rate of the discrete transition, $R_{\mathrm{I}}$ is the ionization rate and $A_{10}$ is the rate of spontaneous emission. For a transition driven by the minor component, $R_{01}=\sigma_{01} F^{-}(r, 0)$ and $R_{\mathrm{I}}=\sigma_{\mathrm{I}} F^{+}(r, 0)$, where $F^{+}(r, 0)$ and $F^{-}(r, 0)$ are the photon fluxes of the major and minor polarization components respectively. In the calculation of the ratio, $F^{-}$is replaced by $F^{+}$ to obtain $V_{I}^{+}\left(M_{J}^{-}\right)$.

In these calculations we have assumed $V_{\text {even }}^{+}=V_{\text {odd }}^{+}$. For the $\Delta J=-1$ transition the good agreement between the calculated and measured values of $\delta^{189}$ Os (see previous discussion) appears to validate this assumption. However, for the $\Delta J=0$ transition and weak focusing, we calculate $r=0.94$, corresponding to only a small isotope selectivity, $\delta^{189} \mathrm{Os} \approx 7 \%$, while the measured isotope shift is much larger, $\delta^{189} \mathrm{Os}=110 \%$. Within the framework discussed above this discrepancy either requires that $V_{\text {odd }}^{+}>V_{\text {even }}^{+}$or suggests that the rate equation approximation is not valid. We emphasize that the model considered in these calculations cannot account for possible quantum mechanical coherence effects and that certain simplifications in the rate equation scheme, i.e. the use of average $V_{\text {even }}^{+}$and $V_{\text {odd }}^{+}$, have been made to facilitate the calculations. 